\title{
Design of an Integrated Vehicle Chassis Control System with Driver Behavior Identification
}

\author{
Bing Zhu, ${ }^{1,2}$ Yizhou Chen, ${ }^{1}$ Jian Zhao, ${ }^{1}$ and Yunfu $\mathrm{Su}^{1}$ \\ ${ }^{1}$ State Key Laboratory of Automotive Simulation and Control, Jilin University, Changchun 130022, China \\ ${ }^{2}$ Key Laboratory of Bionic Engineering of Ministry of Education, Jilin University, Changchun 130022, China \\ Correspondence should be addressed to Jian Zhao; zhaojian@jlu.edu.cn
}

Received 27 April 2015; Accepted 18 August 2015

Academic Editor: Yannis Dimakopoulos

Copyright (c) 2015 Bing Zhu et al. This is an open access article distributed under the Creative Commons Attribution License, which permits unrestricted use, distribution, and reproduction in any medium, provided the original work is properly cited.

An integrated vehicle chassis control strategy with driver behavior identification is introduced in this paper. In order to identify the different types of driver behavior characteristics, a driver behavior signals acquisition system was established using the dSPACE real-time simulation platform, and the driver inputs of 30 test drivers were collected under the double lane change test condition. Then, driver behavior characteristics were analyzed and identified based on the preview optimal curvature model through genetic algorithm and neural network method. Using it as a base, an integrated chassis control strategy with active front steering (AFS) and direct yaw moment control (DYC) considering driver characteristics was established by model predictive control (MPC) method. Finally, simulations were carried out to verify the control strategy by CarSim and MATLAB/Simulink. The results show that the proposed method enables the control system to adjust its parameters according to the driver behavior identification results and the vehicle handling and stability performance are significantly improved.

\section{Introduction}

In recent years, improving the active safety performance of vehicles is what researchers have been working on. With the development of electronic control technology, many active safety systems such as antilock braking system (ABS), traction control system (TCS), electronic stability control (ESC), active front steering (AFS), and four-wheel steering (4WS) have been widely equipped on vehicles to ensure safer and more stable driving experience. However, the potential conflicts among the active control systems increase when they are combined without coordination [1-3]. Thus, there have been plenty of attempts to integrate the chassis control subsystems, for instance, the Integrated Chassis Control (ICC), Unified Chassis Control (UCC), and Vehicle Dynamics Management (VDM) [4-6]. With the implementation of integrated control systems, the interference and coupling among dynamic subsystems are effectively eliminated and the stability performance of vehicle is significantly improved. Nevertheless, integrated control systems nowadays are generally designed in the uniform mode, lacking the consideration of the influence that drivers exert on control systems. As a matter of fact, the driver and the active control system have strong coupling on each other while controlling the vehicle. According to a survey sponsored by National Highway Traffic Safety Administration (NHTSA) of USA, driver's mistake accounts for $90 \%$ or more of all the crashes recorded [7]. Therefore, human driver's characteristics should be involved in the process where the integrated control system is developed.

As the primary control element within the traditional driver-vehicle system, the role of human driver has been studied by a plenty of scientists and researchers. The driver behavior includes driver's sensing, judging, reasoning, deciding, and finally operating the vehicle to turn, accelerate, and brake with strong randomicity, adaptivity, discreteness, and variability. Currently, research is mainly carried out in the aspects of driver behavior modeling, the identification of fatigue, intelligent traffic control, and advanced driver assistance systems (ADAS) [8].

Koh et al. present a tire slip-angle based speed control race driver model through analyzing the vehicle-driver 
interaction at limit handling [9]. Miyajima et al. modeled driver's behavior such as turning the steering wheel or hitting the pedal with Gaussian mixture model (GMM) to identify different kinds of drivers. Compared with the method using raw pedal operation signals, spectral analysis method proposed shows a far better accuracy [10]. Bolia et al. proposed a two-level preview driver steering control model. The outer loop focuses on path following while the inner one tries to capture driver's physical behavior [11]. Lin et al. used several sophisticated artificial neural network architectures to develop driver models in a Driver-Vehicle-Environment (DVE) system [12]. Sathyanarayana et al. proposed a "context and driver aware" (CDA) active vehicle system combining GMM, universal background model, and likelihood maximization based on information fusion to identify driver status and predict driver's distracted behavior [13]. Profound and enlightening works above endeavored to explain what kind of a role driver is playing in a driver-vehicle closedloop system. With further research, early efforts have been made to design the active control system on the basis of driver's characteristics. Macadam studied the physiological limits and physical characteristics of drivers in detail and then established the lateral and longitudinal manipulating model of drivers [14]. Hoult and Cole built a neuromuscular linear model which considers the coactivation of neuromuscular system, muscle, body, and vehicle [15]. Chai et al. described a method to adjust the parameters of steer-by-wire (SBW) system according to the driver's steer characteristics, which could be estimated from experimental data based on the general driver's model [16]. Raksincharoensak et al., Japanese researchers, designed the direct yaw moment control system based on the identification of driver's intention and the performance of the vehicle is significantly improved [17]. In the literature [18], a driver behavior signal capturing system was introduced, based on which the riding comfort and safety of the vehicle are enhanced. Fu et al. integrated a driver model with a run-off-road recovery controller considering driver's target planning, pursuit behavior, compensate behavior, and physical limitations [19]. Keen and Cole proposed a steering controller based on linear model predictive control. A formal system identification procedure is applied to avoid bias from the closed-loop operation of the driver-vehicle system [20]. Although the driver behavior identification methods have made remarkable progress, in practical applications, the driver behavior observation equipment mentioned above is seldom used due to its disadvantages in prize and portability.

The active chassis control systems and the driver have mutual influence for vehicle control with strong couplings. Due to the complexity, polytrope, and uncertainty of driver behavior, it is extremely essential to fully understand the characteristics of drivers and control systems, build the collaborative optimization mechanism, and optimize the performance of the Driver-Vehicle-Environment system.

To design the personalized vehicle control system, one of the most important prerequisites is identifying the driver's individualities. On the basis of the literature [14], Professor Guo proposed a "Preview-Following" system theory, which defines the decision procedure of drivers as preview and compensation. According to this theory, Preview Optimal

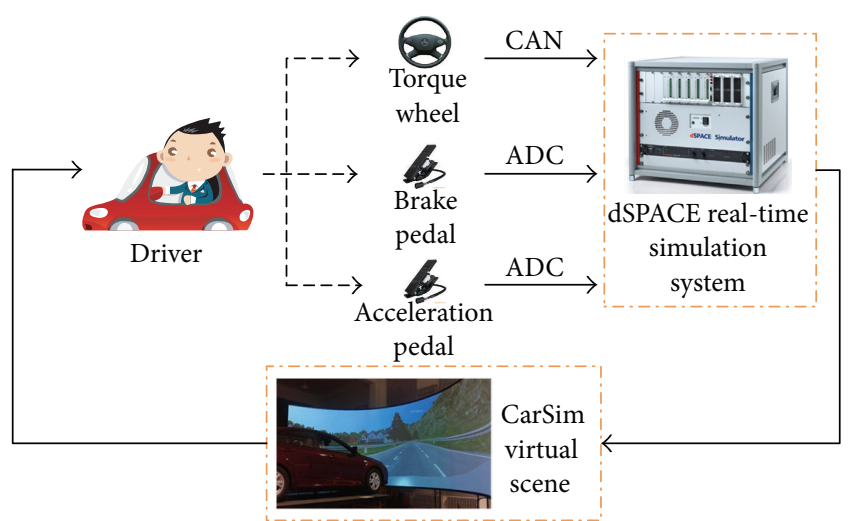

Figure 1: Structure of the driver behavior data acquisition system.

Curvature Model is established. As it demonstrates clearly a relationship between the vehicle stability and driver's characteristics, this theory has been widely used in the research of vehicle stability control and adaptive cruise control due to its high precision and independence of special equipment [21]. Simulation and test results show that this theory precisely depicts driver's steering procedure. The key to applying this theory is the identification of driver's steering characteristics.

In this paper, an integrated chassis control strategy based on the identification of driver characteristics is proposed. First, a real-time driver operation signal acquisition system based on dSPACE is built. Using this system, 10 skilled, 10 normal, and 10 novice drivers' manipulation signals on the same path are collected. Then, genetic algorithm is applied to analyzing the steering characteristics of different kinds of drivers based on the Preview Optimal Curvature Model. An online driver behavior identification method is established using BP neural network. An integrated chassis control (ICC) system with active front steering (AFS) and direct yaw moment control (DYC) is designed using model predictive control (MPC) and considering the driver behavior characteristics. Finally, simulations are carried out to validate the proposed method through the cosimulation of MATLAB/Simulink and CarSim. Test results show that the proposed method enables the control system to adjust its parameters according to the driver behavior identification results and the vehicle stability is effectively enhanced.

\section{Driver Behavior Data Acquisition}

2.1. Driver Behavior Data Acquisition System. In order to precisely analyze the driver behavior, the driver behavior data acquisition system is designed and established based on dSPACE real-time simulation platform, as shown in Figure 1. The dSPACE real-time simulation system could realize the seamless connection to Matlab/Simulink through the automatic code generation and downloading Real-time Interface (RTI). And with the test software ControlDesk, the dSPACE can accomplish the visual management and automatic control of the tests.

In this system, dSPACE DS1006 is used as real-time simulation processor, which collects the opening of accelerator 


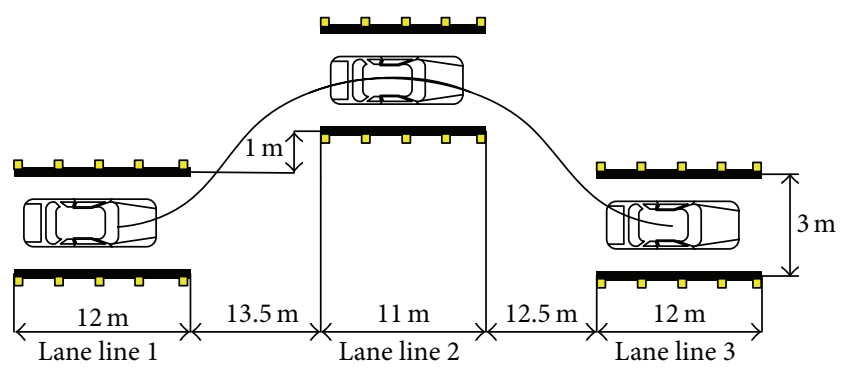

FIGURE 2: Working condition used in driver behavior data acquisition.

pedal and brake pedal along with the steering wheel angel in real-time and meanwhile feeds back the signals to the CarSim vehicle model which runs on an industrial personal computer (IPC). CarSim generates a virtual scene projected to a large screen, according to which the driver perceives the driving environment and operates the vehicle.

In order to enhance the immersion, the torque steering wheel made by SENSODRIVE is adopted in the system. The steering wheel, possessing adjustable variables of damp and friction, can simulate the torque feedback of the real road and greatly enhances the driving experience. The steering wheel is connected to dSPACE DS1006 through CAN bus. The linear potentiometer displacement sensors are used for the acquisition of the accelerator pedal and the brake pedal signals through the AD ports.

2.2. Driver Behavior Data Acquisition. In order to identify the characteristics of different drivers, the usage modes of different kinds of drivers are analyzed. In normal working conditions, the usage modes of different drivers tend to be similar and difficult to distinguish. Thus, driving data acquisition experiments are carried out under the double lane change (DLC) working conditions. The target path is shown in Figure 2.

In the experiments, to guarantee the validation of the test data, 20 male drivers and 10 female drivers are chosen as the test samples. Among the drivers, 10 are skilled drivers, 10 are normal ones, and the last 10 are novice in driving. Before the experiment started, all drivers are trained to be acquainted with the experiment process and the data collection system. When the data collection begins, the driver accelerates from the same start point to the speed of $60 \mathrm{Km} / \mathrm{h}, 80 \mathrm{Km} / \mathrm{h}$, $100 \mathrm{Km} / \mathrm{h}, 110 \mathrm{Km} / \mathrm{h}$, and $120 \mathrm{Km} / \mathrm{h}$, respectively, at the end of lane line 1 . Then, the lane change is carried out according to the target path. When the car passes lane line 3, the driver decelerates the car to stop.

Typical collection signals of three different kinds of drivers are shown in Figure 3. It is seen that, facing the panic lane change, the skilled driver shows shorter response time and could implement lane change smoothly. In contrast, the novice driver tends to operate the vehicle sharply and the vehicle may lose its stability.

\section{Analysis of Driver Behavior Characteristics}

3.1. Preview Optimal Curvature Model. As is shown in Figure 3, among the drivers' operating signals, the steering wheel angles show the most significant difference among three kinds of drivers. Thus, in this research, steering wheel angle is chosen to identify behavior characteristics of different kinds of drivers. Preview optimal curvature model, which is widely used in driver-vehicle closed-loop system research as mentioned above, is introduced here to analyze the driver steering behavior [22].

It is widely believed that the driver plans for the desired path when he/she is driving a vehicle. Assume that the desired path is $Y=f(X)$ and the preview time of driver is a constant, as is shown in Figure 4. At time $t$, the state of the vehicle is

$$
\begin{aligned}
& y=y(t), \\
& \dot{y}=\dot{y}(t) .
\end{aligned}
$$

On the condition that the preview distance is $d$, namely, the driver always gazes at preview point which is $d$ away in front of the vehicle, the preview time could be defined as

$$
T=\frac{d}{V_{x}}
$$

where $V_{x}$ is the longitudinal velocity of the vehicle.

The lateral coordinate of the preview point is $f(t+T)$. If the driver operates a steering angel $\delta_{s w}$, in accordance with which the curvature of the vehicle is $1 / R$, and the lateral acceleration is $\ddot{y}(t)$; in time $T$, the lateral displacement of the vehicle is

$$
f(t+T)=y(t)+T \cdot \dot{y}(t)+\frac{T^{2}}{2} \ddot{y}(t) .
$$

Considering

$$
\ddot{y}=\frac{V_{x}^{2}}{R}=\frac{\delta_{\mathrm{sw}}}{i L} V_{x}^{2},
$$

among which $L$ is the wheel base and $i$ is the steering ratio.

We get the optimal steering wheel angle:

$$
\delta_{\mathrm{sw}}=\frac{2 i L}{d^{2}}(f(t+T)-y(t)-T \cdot \dot{y}(t)) .
$$

Drivers always intend to operate a steering wheel angle $\delta_{\text {sw }}$ under which the displacement of the vehicle could fit the desired path in time $T$. Considering the lag of driver's perception and action together with the dynamic response of the vehicle under a sharp steering, the steering wheel angle $\delta_{\text {sw }}$ that the driver is supposed to operate to track the target path $f$ is

$$
\begin{aligned}
& \frac{\delta_{\mathrm{sw}}}{f}(s) \\
& =\frac{2 C_{0}\left(1+T_{c} s\right) e^{-t_{d} s} s^{2}}{T_{p}^{2}\left(1+T_{h} s\right) s^{2}+2 C_{0}\left(1+T_{c} s\right)\left(1+T_{p} s\right) e^{-t_{d}} s\left(a_{y} / \delta_{\mathrm{sw}}\right)},
\end{aligned}
$$

where $T_{p}$ is the driver's preview time, $t_{d}$ is the driver's neural delay time, $T_{h}$ is the driver's muscle delay time, $T_{c}$ is the correction parameter of steering, and $C_{0}$ is the proportional 


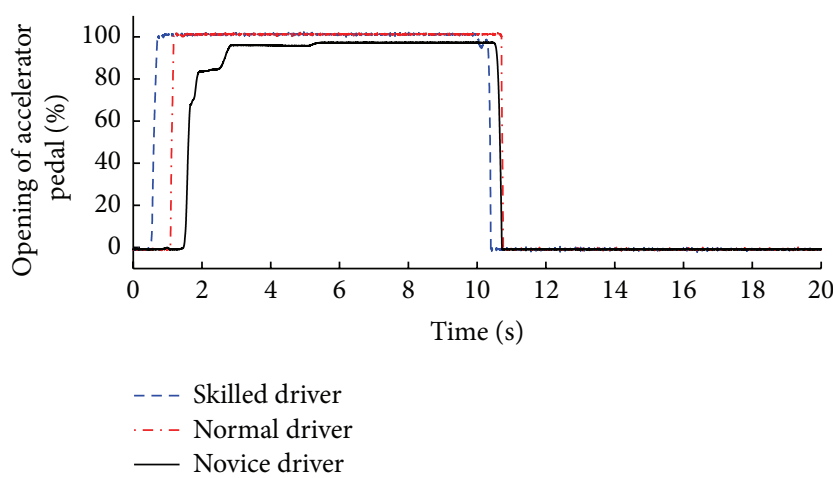

(a) Accelerator pedal input

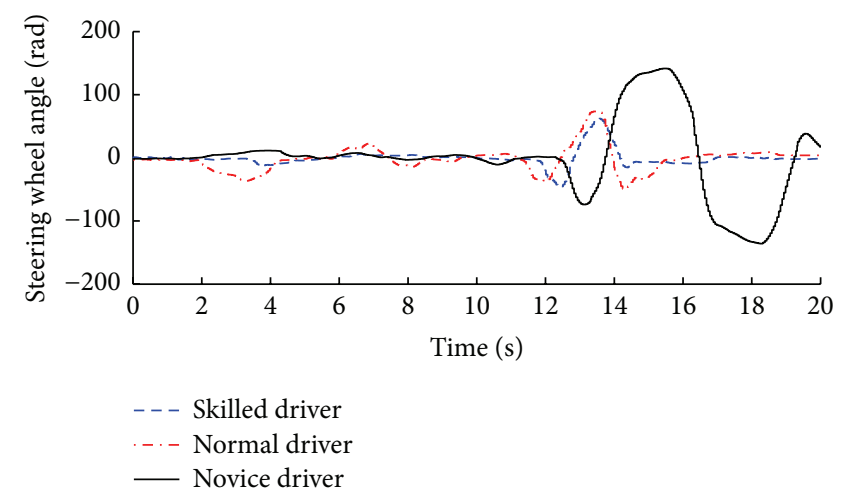

(c) Steering wheel input

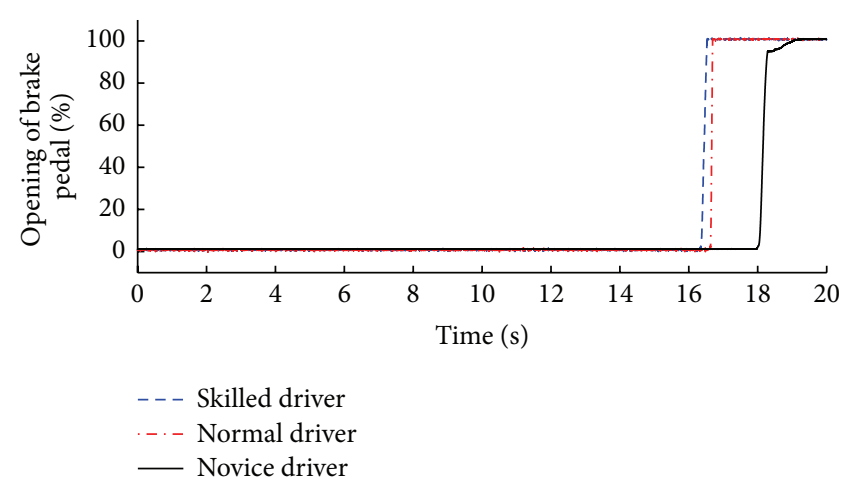

(b) Brake pedal input

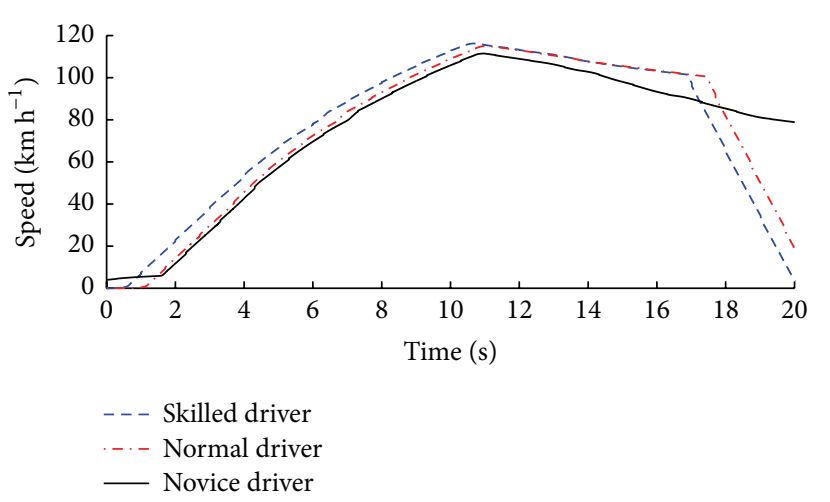

(d) Vehicle speed

Figure 3: Typical signals of different kinds of drivers.

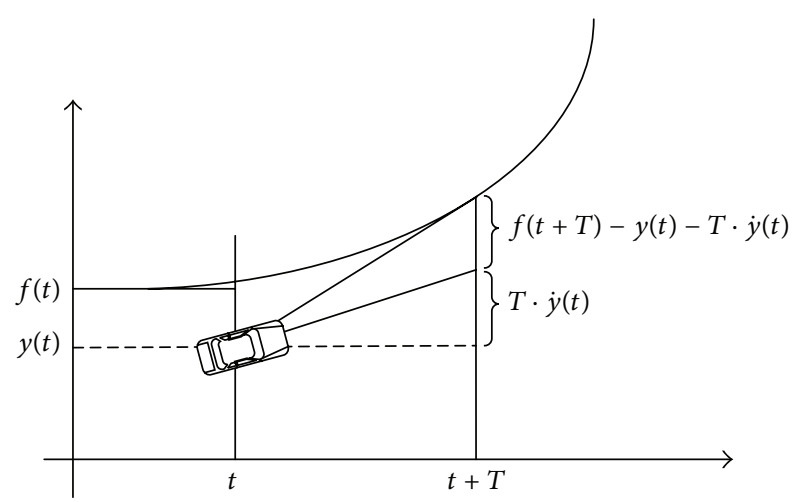

FIgURE 4: Frame of driver's steering decision.

gain correction parameter. The relationships among the parameters are

$$
\begin{aligned}
& T_{c}=t_{d}+T_{h}+T_{a}-\frac{a T_{p}}{3}, \\
& C_{0}=\frac{1}{G_{a_{y}}},
\end{aligned}
$$

where $T_{a}$ is a parameter of the vehicle and $a$ is the following order. $G_{a_{y}}$ is the steady-state gain of lateral acceleration of $a_{y}$ to $\delta_{\text {sw }}$ whose dynamic response is defined as the vehicle model $V(s)$, which will be described in the next section.

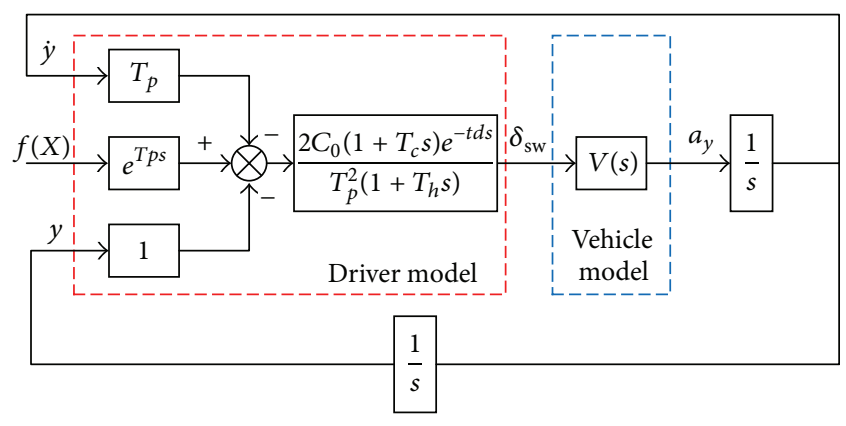

FIGURE 5: Driver preview optimal curvature model.

Therefore, the frame of driver preview optimal curvature model could be demonstrated as in Figure 5, from which it is clearly seen that the parameters $T_{p}, t_{d}$, and $T_{h}$ could characterize different kinds of drivers' behavior, and, namely, the task of identification of different kinds of drivers' steering behavior is confirming the three parameters.

\subsection{Identification of Driver Behavior \\ Characteristics Parameters}

3.2.1. Identification of the Parameters of Vehicle Model. As shown in Figure 5, before identifying the driver's parameters, the characteristics of the vehicle should be specified. 


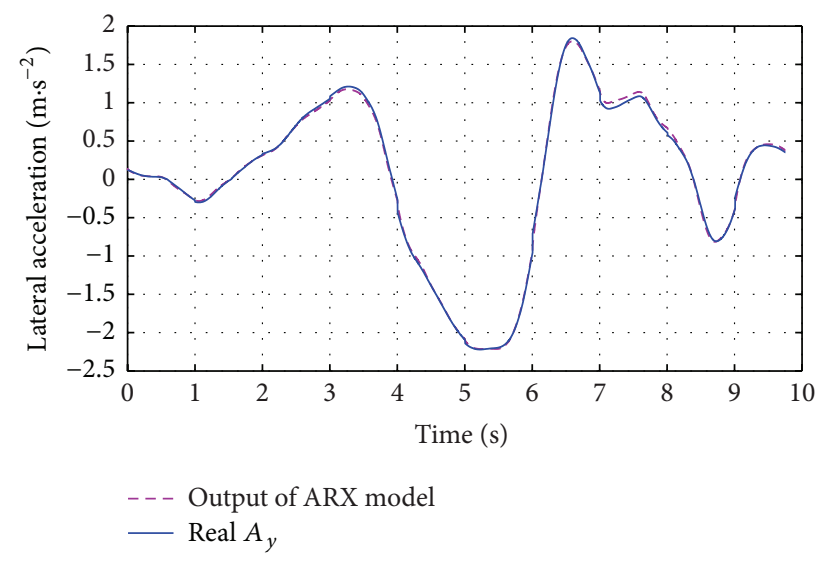

FIgURE 6: Output of vehicle model and real lateral acceleration.

According to the preview optimal curvature model, $V(s)$ could be described as the dynamic response of $a_{y}$ to $\delta_{\text {sw }}$ :

$$
V(s)=\frac{a_{y}}{\delta_{\text {sw }}}(s)=G_{a_{y}} \frac{1+T_{y 1} s+T_{y 2} s^{2}}{1+T_{1} s+T_{2} s^{2}} .
$$

In the ideal situation, $V(s)$ could be directly described as $G_{a_{y}}$. Considering that the vehicle's dynamic response could not be ignored when it is taking a sharp turning, $T_{y 1}, T_{y 2}, T_{1}$, and $T_{2}$ are constants to be identified and $T_{a}$ in (7) is defined as $\left(T_{1}-T_{y 1}\right)$.

The aim of parameters identification is to describe the mathematic relation between the input and output of the system. Since the mechanism of the vehicle dynamic response is not necessarily required in this research, ARX model, which does not demand the explicit physical relationship of the system and works well when dealing with the higherorder system, is adopted here to identify the parameters of the vehicle model. The expression of the ARX model is shown below:

$$
A(z) A_{y}(z)=B(z) u(z)+e(z)
$$

where

$$
\begin{aligned}
& A(z)=1+a_{1} z^{-1}+a_{2} z^{-2}+\cdots+a_{n} z^{-n} \\
& B(z)=1+b_{1} z^{-1}+b_{2} z^{-2}+\cdots+b_{m} z^{-m}
\end{aligned}
$$

Here, $n, m=2, e(z)$ is white noise, $u(z)$ is $\delta_{\text {sw }}$, and $A_{y}(z)$ is $a_{y}$.

Using the MATLAB toolbox to compute, the real lateral acceleration and the output of the identification model are shown in Figure 6 which demonstrates that the vehicle model built here has a high accuracy and the identification rate is $97.2 \%$.

3.2.2. Offline Driver Model Parameters Identification for Different Kinds of Drivers. On the basis of the identification of the vehicle model $V(s)$, driver behavior characteristic parameters, $T_{p}, t_{d}$, and $T_{h}$, are to be identified. Genetic algorithm, which simulates the mechanism of natural selection
TABLE 1: Constraints of driver behavior characteristic parameters.

\begin{tabular}{lcc}
\hline$T_{p}$ & $t_{d}$ & $T_{h}$ \\
\hline$[0.58,2.072]$ & {$[0.17,0.53]$} & {$[0.08,0.36]$} \\
\hline
\end{tabular}

and biological evolutionism, has been widely used in pattern recognition, computing science, automatic control, and so forth. Here, genetic algorithm is adopted to identify the driver model parameters offline.

Firstly, the three model parameters of drivers should be constrained to appropriate scope to guarantee the accuracy of identification results as well as accelerate the identification procedure. As is demonstrated in the literature [22], the parameters are supposed to meet the requirements:

$$
T_{j} \in\left(U_{j \min }, U_{j \max }\right),
$$

where $T_{j}$ is $T_{p}, t_{d}$, and $T_{h} . U_{j \min }$ and $U_{j \max }$ are the minimum and maximum of $T_{p}, t_{d}$, and $T_{h}$, respectively. $j=1,2,3$.

The parameters are constrained to a certain scope shown in Table 1.

To ensure the precision of identification of driver behavior characteristic and that the dynamic responses of the driver model accord well with the experimental data, the target function is defined as the weighted value of the error square sum of steering wheel angel, lateral acceleration, and lateral displacement:

$$
J=w_{1} * J_{1}+w_{2} * J_{2}+w_{3} * J_{3},
$$

where $J_{1}, J_{2}$, and $J_{3}$ are the deviation indices of the steering wheel angel, the lateral acceleration, and the lateral displacement, respectively. Their expressions are shown as

$$
\begin{aligned}
& J_{1}=\frac{1}{t_{0}} \int_{0}^{t_{0}}\left(\delta_{\mathrm{sw}}-\widehat{\delta}_{\mathrm{sw}}\right)^{2} d t, \\
& J_{2}=\frac{1}{t_{0}} \int_{0}^{t_{0}}\left(a_{y}-\widehat{a}_{y}\right)^{2} d t, \\
& J_{3}=\frac{1}{t_{0}} \int_{0}^{t_{0}}(y-\widehat{y})^{2} d t,
\end{aligned}
$$

where $\widehat{\delta}_{\mathrm{sw}}, \hat{a}_{y}$, and $\widehat{y}$ are the theoretical data based on driver model and vehicle model. $w_{1}, w_{2}$, and $w_{3}$ are weight coefficients and $t_{0}$ is the sampling time.

Since there are three variables, the fitness function is determined according to the simple adaptive function method. Besides, the binary encoding method, proportion selection strategy, single point switching strategy, and simple mutation strategy are adopted [23].

As mentioned in Section 2, the drivers can be divided into three categories, the skilled ones, normal ones, and novice ones. With genetic algorithm, the driver's characteristics could be identified quantitatively and the results of the identification of parameters are listed in Table 2.

When the offline identification is finished, the results are adopted as the training output of the online BP neural network model used in online characteristics identification. 
TABLE 2: Identification results of driver behavior characteristic parameters.

\begin{tabular}{lccc}
\hline Driver & Average & Standard deviation & Target variance \\
\hline Novice drivers & & & \\
$T_{p}$ & 0.7100 & 0.0964 & \\
$t_{d}$ & 0.1489 & 0.0834 & $4.7016 e-003$ \\
$T_{h}$ & 0.3740 & 0.0637 & \\
\hline Skilled drivers & & & \\
$T_{p}$ & 1.6954 & 0.0961 & \\
$t_{d}$ & 0.3763 & 0.0720 & $5.5144 e-003$ \\
$T_{h}$ & 0.3632 & 0.0560 & \\
\hline Normal drivers & & & \\
$T_{p}$ & 1.1534 & 0.1015 & \\
$t_{d}$ & 0.2388 & 0.1132 & \\
$T_{h}$ & 0.2577 & 0.0873 & \\
\hline
\end{tabular}

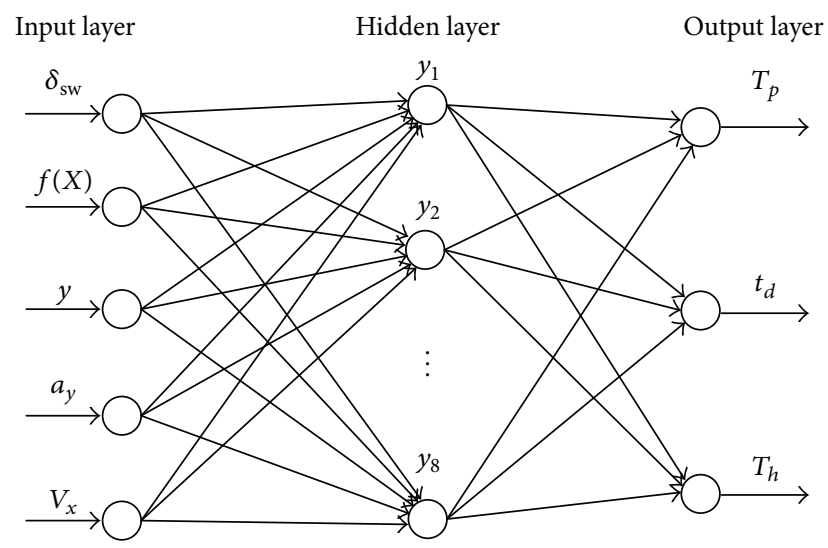

Figure 7: Structure of the BP neural network model.

3.2.3. Online Driver Behavior Characteristic Parameters Identification. Due to the genetic algorithm's relative timeconsuming, low efficiency, and absence of meeting the realtime requirement of the control system, in this research, BP neural network algorithm is designed for online identification of driver behavior characteristic parameters. BP neural network is established as in Figure 7.

The BP neural network model has 3 layers; the number of nodes of the input layer, hidden layer, and output layer $n, l$, and $m$ is 5,8 , and 3 , respectively. The five nodes $x_{1} \sim x_{5}$ in input layer are $\delta_{\mathrm{sw}}, f, y, a_{y}$, and $V_{x}$, respectively, $\delta_{\mathrm{sw}}$ is driver's steering wheel angle, $f$ is the lateral displacement of the target path, $y$ is the real lateral displacement of the vehicle, $a_{y}$ is the lateral acceleration of the vehicle, and $V_{x}$ is the longitudinal velocity of the vehicle. The three nodes $z_{1} \sim z_{3}$ in output layer are $T_{p}, t_{d}$, and $T_{h}$, which are the drive's behavior characteristics to be identified.

The output of the hidden layer is

$$
H_{j}=g\left(\sum_{i=1}^{n} \omega_{i j} x_{i}-h_{j}\right), \quad j=1,2, \ldots, l
$$

The output of the output layer is

$$
O_{k}=\sum_{j=1}^{l} \omega_{j k} H_{j}-o_{k}, \quad k=1,2, \ldots, m,
$$

where $\omega_{i j}$ and $\omega_{j k}$ are weighting coefficients of input layer to hidden layer and hidden layer to output layer, respectively. The thresholds of the hidden layer and output layer are $h$ and $o$, respectively. The $g(x)$ is the excitation function:

$$
g(x)=\frac{1}{1+e^{-x}} .
$$

The deviation calculation function is used to calculate the deviation of the desired output and the calculated output of the model:

$$
e_{k}=\frac{1}{2}\left(Y_{k}-O_{k}\right)^{2}, \quad k=1,2, \ldots, m,
$$

where $Y_{k}$ is the desired output.

In order to minimize the variance of the output, backpropagation modification weight matrix is used as the learning rule of the model:

$$
\begin{aligned}
& \omega_{i j}^{\text {new }}=\omega_{i j}+\eta H_{j}\left(1-H_{j}\right) x(i) \sum_{k=1}^{m} \omega_{j k} e_{k} \\
& i=1,2, \ldots, n ; j=1,2, \ldots, l, \\
& \omega_{j k}^{\text {new }}=\omega_{j k}+\eta H_{j} e_{k}, \quad j=1,2, \ldots, l ; k=1,2, \ldots, m \\
& h_{j}^{\text {new }}=h_{j}+\eta H_{j}\left(1-H_{j}\right) \sum_{k=1}^{m} \omega_{j k} e_{k}, \quad j=1,2, \ldots, l \\
& o_{k}^{\text {new }}=o_{k}+e_{k}, \quad k=1,2, \ldots, m,
\end{aligned}
$$

where $\omega_{i j}^{\text {new }}$ and $\omega_{j k}^{\text {new }}$ are weighting coefficients after update and $h_{j}^{\text {new }}$ and $o_{k}^{\text {new }}$ are thresholds after update. $\eta$ is the learning rate.

The offline identification results obtained from genetic algorithm are used to train the BP neural network model. And when the training performance is as shown in Figure 8, the training is stopped. The datasheet trained from neural network model could be directly used as driver behavior characteristic parameters identification model. Therefore, the driver model whose characteristics fit the current driver's behavior is achieved.

Thus, when a driver is driving a car, his/her behavior characteristic parameters could be identified rapidly, on the basis of which the control system could be designed to fit the driver's characteristics.

\section{Design of Integrated Chassis Control System}

The architecture of the proposed ICC system for AFS and DYC integration with driver behavior identification is shown in Figure 9. The control system is mainly consisted of driver identification module, preview optimal curvature driver model, 2-Degree-of-Freedom (DOF) vehicle reference 


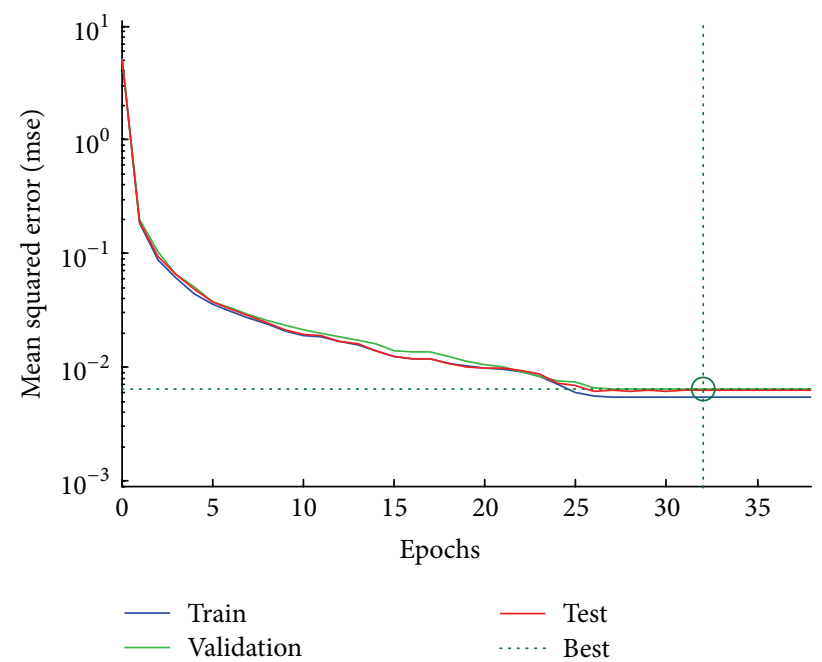

Figure 8: Performance of neural network model.

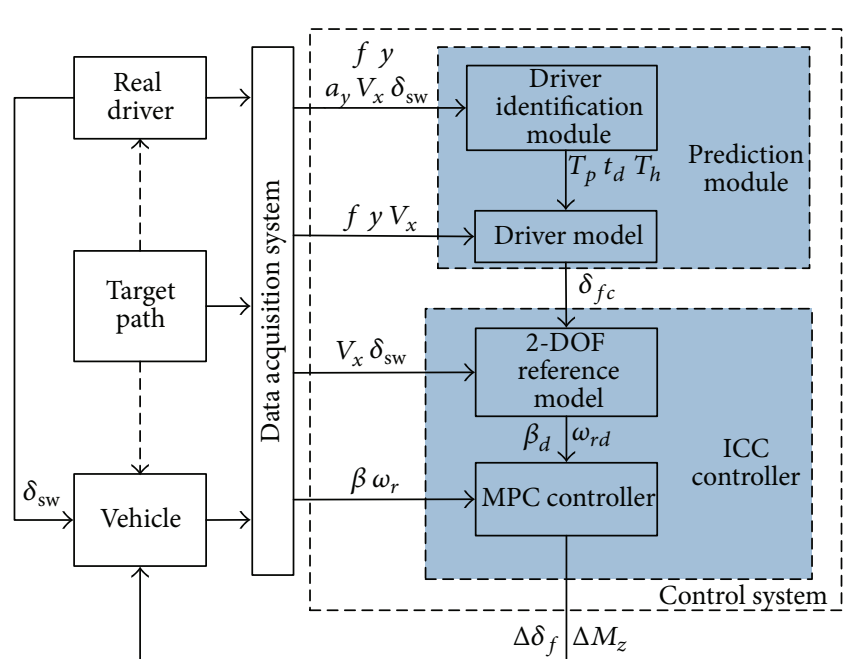

FIGURE 9: ICC system configurations with driver behavior identification.

model, and model prediction control (MPC) based controller module. Different kinds of drivers' characteristics are marked by the three parameters $T_{p}, t_{d}$, and $T_{h}$ as is shown in Section 3; the parameters $T_{p}, t_{d}$, and $T_{h}$ are identified online according to the driver's operation and the state of the vehicle. When the controller is on, the three parameters are used to build the driver model. $\delta_{f c}$ is defined as predicted front wheel angle based on driver model. $\delta_{f c}$ of the certain driver could be predicted at next sample time according to the identification result along with vehicle motion state. Based on the predicted $\delta_{f c}, 2$-DOF reference model could output the desired yaw rate and side slip angle which are the target of the MPC controller. The MPC controller optimizes online the active control front wheel steering angle $\Delta \delta_{f}$ and yaw moment $\Delta M_{z}$.

4.1. Linear 2-DOF Reference Model. The 2-DOF reference vehicle model which considers both accuracy and simplicity is used for target outputs calculation, as shown in Figure 10.

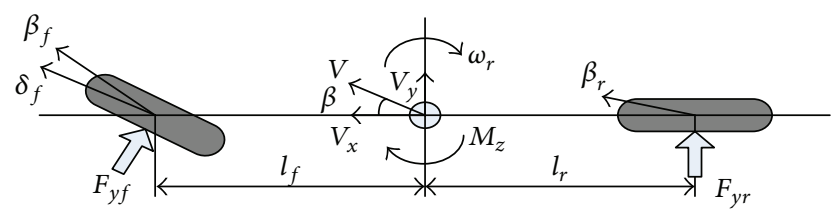

Figure 10: 2-DOF reference model.

The side-slip angle $\beta$ and yaw rate $\omega_{r}$ are described in the model.

The vehicle state space equation is

$$
\begin{aligned}
& \dot{x}=\mathbf{A}_{0} x+\mathbf{B}_{0} u, \\
& y=\mathbf{C}_{0} x,
\end{aligned}
$$

where

$$
\begin{aligned}
x & =\left[\begin{array}{ll}
\beta & \omega_{r}
\end{array}\right]^{\mathrm{T}}, \\
u & =\left[\begin{array}{ll}
\delta_{f} & M_{Z}
\end{array}\right]^{\mathrm{T}}, \\
\mathbf{A}_{0} & =\left[\begin{array}{ll}
a_{11} & a_{12} \\
a_{21} & a_{22}
\end{array}\right]=\left[\begin{array}{cc}
-\frac{c_{f}+c_{r}}{m \cdot V_{x}} & -1+\frac{c_{r} l_{r}-c_{f} l_{f}}{m \cdot V_{x}^{2}} \\
\frac{c_{r} l_{r}-c_{f} l_{f}}{I_{z}} & -\frac{c_{r} l_{r}^{2}+c_{f} l_{f}^{2}}{I_{z} \cdot V_{x}}
\end{array}\right], \\
\mathbf{B}_{0} & =\left[\begin{array}{ll}
b_{11} & b_{12} \\
b_{21} & b_{22}
\end{array}\right]=\left[\begin{array}{ll}
\frac{c_{f}}{m \cdot V_{x}} & 0 \\
\frac{c_{f} l_{f}}{I_{z}} & \frac{1}{I_{z}}
\end{array}\right], \\
\mathbf{C}_{0} & =\left[\begin{array}{ll}
c_{11} & c_{12} \\
c_{21} & c_{22}
\end{array}\right]=\left[\begin{array}{ll}
1 & 0 \\
0 & 1
\end{array}\right],
\end{aligned}
$$

where $c_{f}, c_{r}$ are front and rear axle cornering stiffness; $m$ is mass; $l_{f}, l_{r}$ describe the distances from the vehicle CG to the front and rear axle, respectively; $I_{z}$ is yaw inertia of the vehicle; and $\delta_{f}$ and $M_{z}$ are the front wheel steering angle and yaw moment of the vehicle.

To guarantee the lateral stability of the vehicle, the yaw rate should be restricted within a stable field. The desired yaw rate could be obtained from the steady-state gain of the yaw rate of the reference model:

$$
\omega_{r d}=\frac{V_{x} \cdot \delta_{f}}{\left(l_{f}+l_{r}\right) \cdot\left[1+\left(V_{x} / v_{\mathrm{ch}}\right)^{2}\right]},
$$

where

$$
v_{\mathrm{ch}}^{2}=\frac{c_{f} \cdot c_{r} \cdot\left(l_{f}+l_{r}\right)^{2}}{m\left(c_{r} l_{r}-c_{f} l_{f}\right)} .
$$

Also, the desired yaw rate should be restricted according to the road friction coefficient $\mu$ :

$$
\left|\omega_{r d}\right| \leq \mu \cdot \frac{g}{V_{x}} .
$$


To maintain lateral stability, it is important to sustain driver's control authority, which can be achieved when the vehicle sideslip angle is small. According to some literatures [24], the desired sideslip angle can be chosen as

$$
\beta_{d}=0
$$

4.2. Design of Integrated Chassis Controller Based on MPC. As a novel methodology with prediction of future states to minimize the deviation between the ideal and actual output of the system, the model predictive control (MPC) shows good stability and robust performance [20,25-27]. So it draws attentions by the design of integrated chassis control system with consideration of driver behaviors.

In this paper, the actual and expected side slip angle and yaw rate are selected as the inputs of the MPC controller, and the outputs are expected active yaw moment and front steering angle. In the controller, the predictive model based on the 2-DOF reference model is built to predict the controlled output firstly. Then, the quadratic programming is developed for the optimal solution.

First, the state space function (21) is discretized as

$$
\begin{aligned}
x(k+1) & =A x(k)+B u(k), \\
y(k) & =C x(k) .
\end{aligned}
$$

And the increment equation could be written as

$$
\begin{aligned}
\Delta x(k+1) & =A \Delta x(k)+B \Delta u(k), \\
y(k) & =C \Delta x(k)+y(k-1),
\end{aligned}
$$

where

$$
\begin{aligned}
& \Delta x(k)=x(k)-x(k-1) \\
& \Delta u(k)=u(k)-u(k-1) .
\end{aligned}
$$

$A=I+T_{s} A_{0} ; B=T_{s} B_{0}$; and $C=C_{0}$, where $T_{s}$ is the discrete sampling time.

Then the predictive controlled output is

$$
Y(k+1 \mid k)=S \Delta x(k)+E y(k)+F \Delta U(k),
$$

where

$$
\begin{gathered}
Y(k+1 \mid k)=\left[\begin{array}{c}
y(k+1 \mid k) \\
y(k+2 \mid k) \\
\vdots \\
y(k+p \mid k)
\end{array}\right], \\
\Delta U(k)=\left[\begin{array}{c}
\Delta u(k) \\
\Delta u(k+1) \\
\vdots \\
\Delta u(k+c-1)
\end{array}\right],
\end{gathered}
$$$$
S=\left[\begin{array}{c}
C A \\
C A^{2}+C A \\
\vdots \\
\sum_{i=1}^{p} C A^{i}
\end{array}\right]
$$$$
F=\left[\begin{array}{ccccc}
C B & 0 & 0 & \cdots & 0 \\
\sum_{i=1}^{2} C A^{i-1} B & C B & 0 & \cdots & 0 \\
\vdots & \vdots & \vdots & \cdots & \vdots \\
\sum_{i=1}^{C} C A^{i-1} B & \sum_{i=1}^{C-1} C A^{i-1} B & \cdots & C B \\
\vdots & \vdots & \vdots & \cdots & \vdots \\
\sum_{i=1}^{P} C A^{i-1} B & \sum_{i=1}^{P-1} C A^{i-1} B & & \cdots & \sum_{i=1}^{P-C+1} C A^{i-1} B
\end{array}\right],
$$$$
E=\left[\begin{array}{c}
I \\
I \\
\vdots \\
I
\end{array}\right],
$$

where $p$ and $c$ are the predictive and control horizons, respectively, and $c \leq p$.

The cost function could be described as

$$
\begin{aligned}
J(y(k), \Delta U(k))= & \left\|\lambda_{y}\left(Y(k+1 \mid k)-Y_{d}(k+1)\right)\right\|^{2} \\
& +\left\|\lambda_{u} \Delta U(k)\right\|^{2},
\end{aligned}
$$

where $\lambda_{y}$ is the weight coefficient of the output deviation, $\lambda_{u}$ is the weight coefficient of control input increment, and $Y_{d}(k+$ 1 ) is the desired output in the prediction horizon; namely,

$$
Y_{d}(k+1)=\left[\begin{array}{ll}
\beta_{d} & \omega_{r d}
\end{array}\right]^{\mathrm{T}} .
$$

At each time step the following optimization problem is solved:

$$
\min _{\Delta U(k)} J(y(k), \Delta U(k)) .
$$

With the constraints of the inputs, the variation of inputs and the outputs, respectively, is

$$
\begin{gathered}
u_{\min }(k+j) \leq u(k+j) \leq u_{\max }(k+j), \\
j=0,1, \ldots, c-1, \\
-\Delta u_{\min }(k+j) \leq \Delta u(k+j) \leq \Delta u_{\max }(k+j), \\
j=0,1, \ldots, c-1, \\
y_{\text {min }}(k+j) \leq y(k+j) \leq y_{\max }(k+j), \\
j=0,1, \ldots, p-1,
\end{gathered}
$$


TABLE 3: Vehicle parameters for simulation.

\begin{tabular}{lcc}
\hline Parameters & Symbol & Values \\
\hline Mass (kg) & $m$ & 2210 \\
Front axle cornering stiffness (N/rad) & $c_{f}$ & 62800 \\
Rear axle cornering stiffness (N/rad) & $c_{r}$ & 68000 \\
Distance from vehicle CG to the front axle (m) & $l_{f}$ & 1.07 \\
Distance from vehicle CG to the rear axle $(\mathrm{m})$ & $l_{r}$ & 2.23 \\
Vehicle moment of inertia about the $z$-axis $\left(\mathrm{kgm}^{2}\right)$ & $I_{z}$ & 4331.6 \\
\hline
\end{tabular}

where inequalities (34) limit the control inputs and (35) constrain the changes of the control input, while (36) are constraints on system output variables.

Obviously, it is a typical constraint optimization problem and can be transformed into a quadratic programming (QP) problem:

$$
\begin{array}{ll}
\min _{\Delta U(k)} & \frac{1}{2} \Delta U(k)^{T} W_{H} \Delta U(k)+W_{G}^{T} \Delta U(k) \\
\text { s.t. } & A_{\text {const }} \Delta U(k) \geq b_{\text {const }},
\end{array}
$$

where

$$
\begin{gathered}
W_{H}=2\left(S^{T} \lambda_{y}^{T} \lambda_{y} S+\lambda_{u}^{T} \lambda_{u}\right) \\
W_{G}=-2 S^{T} \lambda_{y}^{T} \lambda_{y} E_{p}(k+1 \mid k) \\
E_{p}(k+1 \mid k)=Y_{d}(k+1)-S \Delta x(k)-E y(k),
\end{gathered}
$$

where $A_{\text {const }}$ and $b_{\text {const }}$ are the constrained matrices.

Then, the first sample of the results is used to compute the optimal steering angle and direct yaw moment by the following feedback control law:

$$
\begin{aligned}
\Delta u(k) & =\left[\begin{array}{cc|ccc}
1 & 0 & 0 & \cdots & 0 \\
0 & 1 & 0 & \cdots & 0
\end{array}\right]_{2 \times 2 C} \Delta U(k), \\
u(k) & =u(k-1)+\Delta u(k) .
\end{aligned}
$$

\section{Analysis and Simulation}

In order to verify the integrated vehicle chassis control strategy based on driver behavior identification, the cosimulation of MATLAB/Simulink and CarSim is adopted, and the primary parameters of vehicle are shown in Table 3.

The simulation is carried out under a typical double lane change working condition. The driver model in CarSim is used and its characteristic is identified by the algorithm online. To verify the robust performance of the control strategy when the speed varies, the simulation is started at the longitudinal speed of $60 \mathrm{Km} / \mathrm{h}$ and $80 \mathrm{Km} / \mathrm{h}$, respectively.

According to the online identification results, the three steering parameters of driver model in CarSim are shown in Table 4.

The three parameters are adopted in the preview optimal curvature driver model built in MATLAB/Simulink. The test results of the control strategy are shown in Figures 11-12, in which (a) is the trajectories, (b) and (c) are yaw rate and lateral
TABLE 4: Identification results of driver behavior characteristic parameters.

\begin{tabular}{lcc}
\hline$T_{p}$ & $t_{d}$ & $T_{h}$ \\
\hline 1.3886 & 0.4176 & 0.1589 \\
\hline
\end{tabular}

acceleration respectively, and (d) and (e) are the controlled steering angle and direct yaw moment, respectively. It is seen that both vehicles can track the target path acceptably; moreover, the path-following performance of the vehicle is clearly further improved by the usage of driver behavior identification (DBI) and MPC compared to the vehicle with only MPC. In the meanwhile, both the yaw rate and the lateral acceleration of the vehicle with DBI and MPC track the desired value more closely than vehicle with only MPC, which indicates that the handling and stability are further improved by the introduction of DBI into MPC. Furthermore, both actuators execute reduced output magnitude. It is implied that their energy consumption are optimized compared to the vehicle equipped with only MPC controller; besides, as normally implemented by active braking control, a reduced direct yaw moment control will also lessen the losses of vehicle speed and engine power.

Therefore, on double lane change, the integrated chassis control system based on MPC can effectively ensure the vehicle driving stability. When combined with DBI, the integrated controller further regulates the lateral displacement, yaw rate, and lateral acceleration to a desired region with reduced output of actuators.

\section{Conclusion}

This paper presents an integrated control strategy with driver behavior identification.

Firstly, the driver behavior data acquisition system is designed and established based on dSPACE real-time simulation platform, and the driver inputs of different kinds of drivers were collected under the double lane change test condition. As is shown in collected data, among the drivers' operating signals, the steering wheel angles show the most significant difference among different kinds of drivers.

Based on this, preview optimal curvature model is introduced to analyze the driver steering behavior. The genetic algorithm for offline usage and a neural network algorithm for online usage are designed to recognize different patterns of drivers, specifically, to identify the three steering parameters of driver model.

Then, an integrated control strategy for active steering angle and direct yaw moment is proposed. The identified driver model is used to predict the steering angle next sample time according to the target path and vehicle state. A linear 2DOF reference model is adopted to calculate the desired state of the vehicle and model predictive control (MPC) is used to calculate the active control quantity to be exerted on vehicle.

Finally, simulations are carried out with the cosimulation of MATLAB/Simulink and CarSim. The results indicate that the proposed method could identify the driver behavior characteristic parameters and enable the control system to adjust 

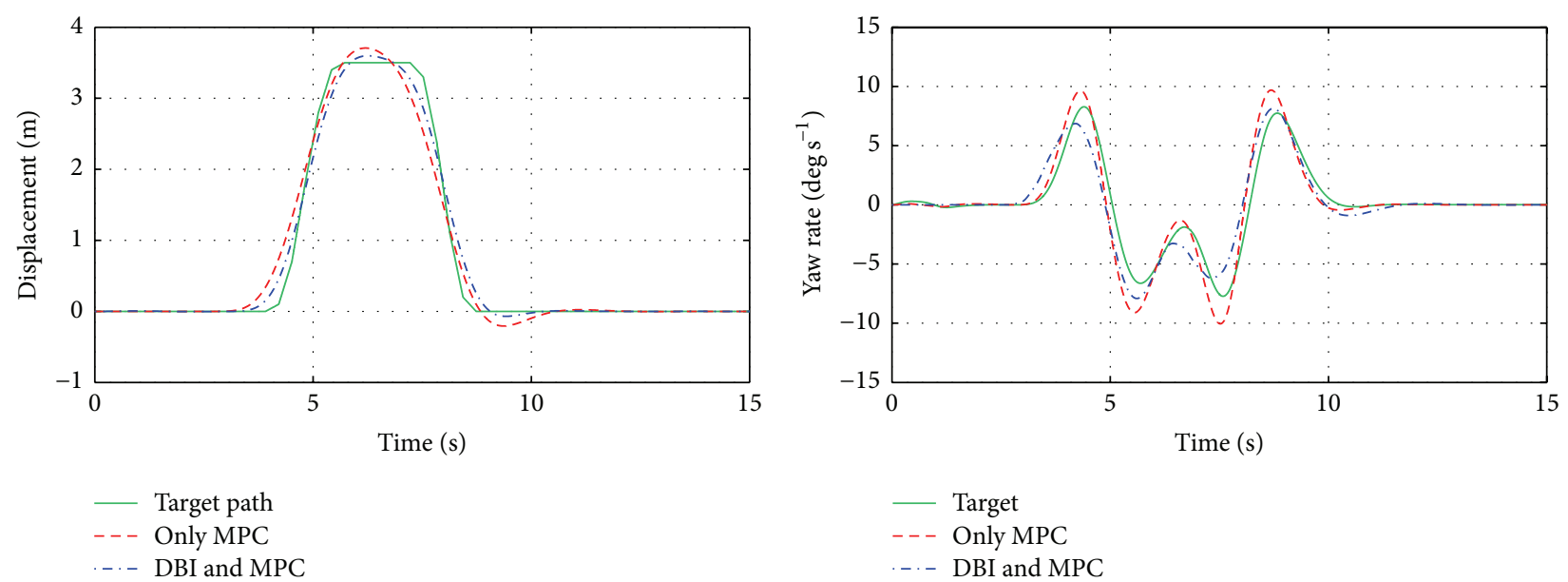

(a)

(b)
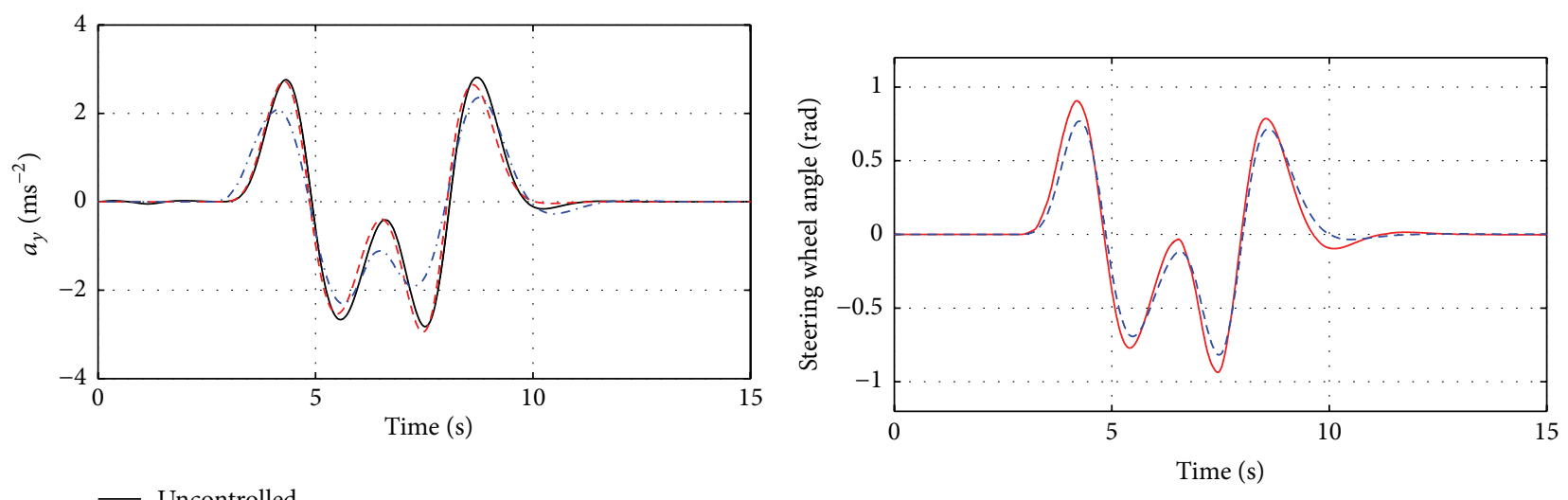

- Uncontrolled

- - - Only MPC

... DBI and MPC

- Only MPC

- - DBI and MPC

(c)

(d)

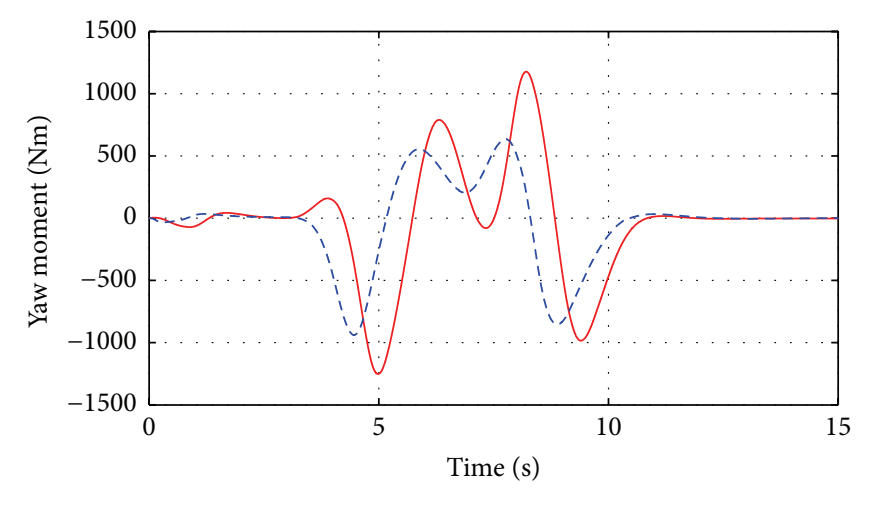

- Only MPC

- - DBI and MPC

(e)

Figure 11: Test results at speed of $60 \mathrm{Km} / \mathrm{h}$. 

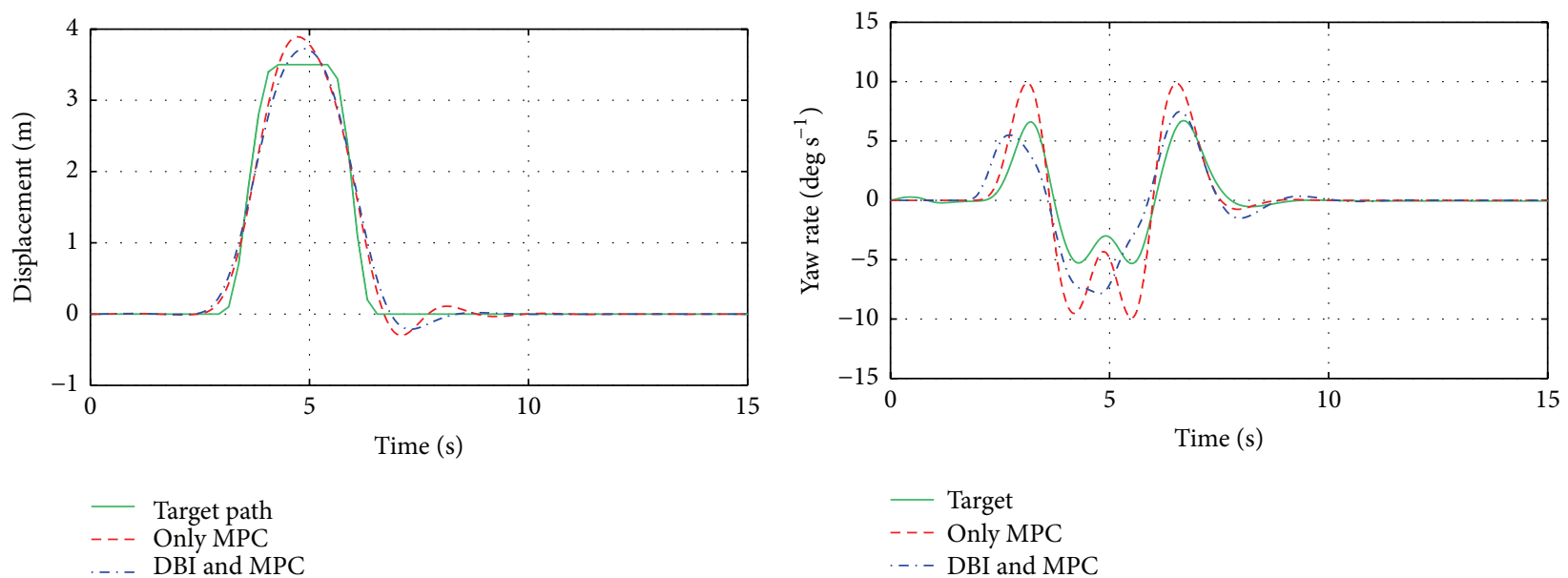

(a)

(b)
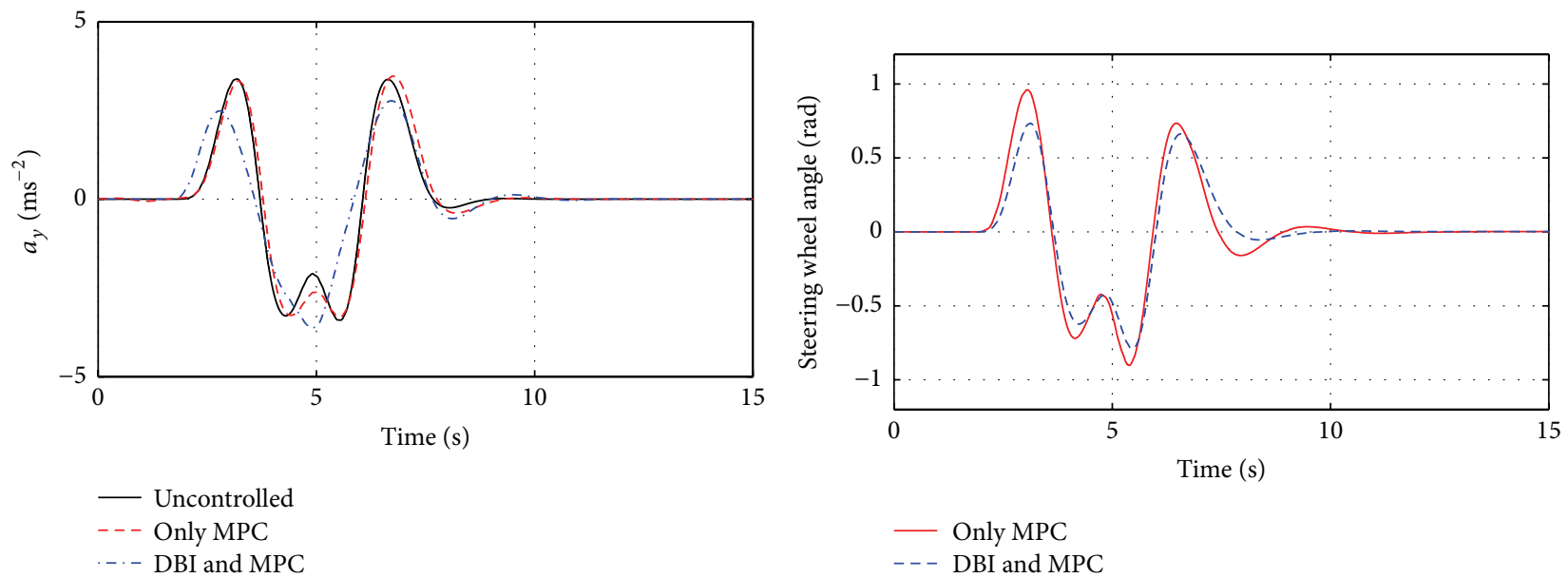

(c)

(d)

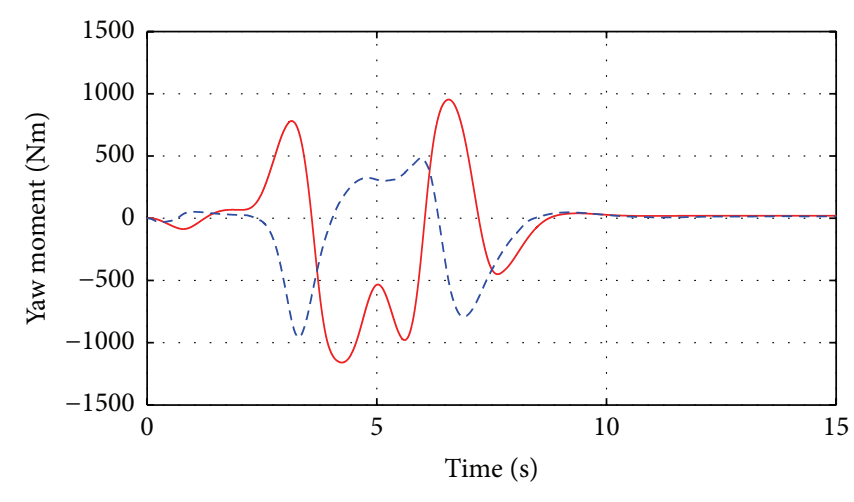

- Only MPC

- - DBI and MPC

(e)

Figure 12: Test results at speed of $80 \mathrm{Km} / \mathrm{h}$. 
its parameters according to the driver behavior identification results. Meanwhile, the integrated control considering the driver's characteristics further enhanced the vehicle's handling and stability performance compared to individual MPC control.

\section{Conflict of Interests}

The authors declare that there is no conflict of interests regarding the publication of this paper.

\section{Acknowledgments}

This work is partially supported by the National Natural Science Foundation of China (51105169, 51205156, and 51475206) and Jilin Province Science and Technology Development Plan Projects (20140204010GX).

\section{References}

[1] F. Yu, D.-F. Li, and D. A. Crolla, "Integrated vehicle dynamics control-state-of-the art review," in Proceedings of the IEEE Vehicle Power and Propulsion Conference (VPPC '08), Harbin, China, September 2008.

[2] Y. Kou, H. Peng, and D. Jung, "Development and evaluation of an integrated chassis control system," JSAE Review of Automotive Engineering, vol. 29, no. 3, 2008.

[3] A. Elmarakbi, C. Rengaraj, A. Wheately, and M. Elkady, "New integrated chassis control systems for vehicle handling performance enhancement," International Journal of Dynamics and Control, vol. 1, no. 4, pp. 360-384, 2013.

[4] C. Rengaraj and D. Crolla, "Integrated chassis control to improve vehicle handling dynamics performance," SAE Paper 2011-01-0958, 2011.

[5] W. Cho, J. Yoon, J. Kim, J. Hur, and K. Yi, "An investigation into unified chassis control scheme for optimised vehicle stability and manoeuvrability," Vehicle System Dynamics, vol. 46, no. 1, pp. 87-105, 2008.

[6] A. Trächtler, "Integrated vehicle dynamics control using active brake, steering and suspension systems," International Journal of Vehicle Design, vol. 36, no. 1, pp. 1-12, 2004.

[7] J. C. Fell and M. Freedman, The Relative Frequency of Unsafe Driving Acts in Serious Traffic Crashes, National Highway Traffic Safety Administration, Ishington, DC, USA, 2001.

[8] T. Toledo, "Driving behaviour: models and challenges," Transport Reviews, vol. 27, no. 1, pp. 65-84, 2007.

[9] Y. Koh, K. Yi, and K. Kim, "A tire slip-angle based speed control driver model for analysis of vehicle-driver systems at limit handling," SAE Technical Paper 2015-01-1566, 2015.

[10] C. Miyajima, Y. Nishiwaki, K. Ozawa et al., "Driver modeling based on driving behavior and its evaluation in driver identification," Proceedings of the IEEE, vol. 95, no. 2, pp. 427-437, 2007.

[11] P. Bolia, T. Weiskircher, and S. Müller, "Driver steering model for closed-loop steering function analysis," Vehicle System Dynamics, vol. 52, no. 1, pp. 16-30, 2014.

[12] Y. Lin, P. Tang, W. J. Zhang, and Q. Yu, "Artificial neural network modelling of driver handling behaviour in a driver-vehicleenvironment system," International Journal of Vehicle Design, vol. 37, no. 1, pp. 24-45, 2005.
[13] A. Sathyanarayana, P. Boyraz, and J. H. L. Hansen, "Information fusion for robust 'context and driver aware' active vehicle safety systems," Information Fusion, vol. 12, no. 4, pp. 293-303, 2011.

[14] C. C. Macadam, "Understanding and modeling the human driver," Vehicle System Dynamics, vol. 40, no. 1-3, pp. 101-134, 2003.

[15] W. Hoult and D. J. Cole, "A neuromuscular model featuring co-activation for use in driver simulation," Vehicle System Dynamics, vol. 46, no. 1, pp. 175-189, 2008.

[16] Y. W. Chai, Y. Abe, Y. Kano, and M. Abe, "A study on adaptation of SBW parameters to individual driver's steer characteristics for improved driver-vehicle system performance," Vehicle System Dynamics, vol. 44, supplement 1, pp. 874-882, 2006.

[17] P. Raksincharoensak, T. Mizushima, and M. Nagai, "Direct yaw moment control system based on driver behaviour recognition," Vehicle System Dynamics, vol. 46, supplement 1, pp. 911-921, 2008.

[18] A. Sathyanarayana, P. Boyraz, Z. Purohit, R. Lubag, and J. H. L. Hansen, "Driver adaptive and context aware active safety systems using CAN-bus signals," in Proceedings of the IEEE Intelligent Vehicles Symposium (IV '10), pp. 1236-1241, IEEE, June 2010.

[19] C. Fu, P. T. Freeman, and J. R. Wagner, "Driver models for virtual testing of automotive run-off-road and recovery control systems and education strategies," SAE Paper 2015-01-0256, 2015.

[20] S. D. Keen and D. J. Cole, "Bias-free identification of a linear model-predictive steering controller from measured driver steering behavior," IEEE Transactions on Systems, Man, and Cybernetics, Part B: Cybernetics, vol. 42, no. 2, pp. 434-443, 2012.

[21] A. Y. Ungoren and H. Peng, "An adaptive lateral preview driver model," Vehicle System Dynamics, vol. 43, no. 4, pp. 245-259, 2005.

[22] K. Guo, F. Ma, and F. Kong, "Driver model identification of driver-vehicle-road closed-loop system," Automotive Engineering, vol. 24, no. 1, 2002.

[23] L. Davis, Ed., Handbook of Genetic Algorithms, vol. 115, Van Nostrand Reinhold, New York, NY, USA, 1991.

[24] X. Shen and F. Yu, "Investigation on integrated vehicle chassis control based on vertical and lateral tyre behaviour correlativity," Vehicle System Dynamics, vol. 44, no. 1, pp. 506-519, 2006.

[25] D. Q. Mayne, J. B. Rawlings, C. V. Rao, and P. O. Scokaert, "Constrained model predictive control: stability and optimality," Automatica, vol. 36, no. 6, pp. 789-814, 2000.

[26] T. Qu, H. Chen, Y. Ji, H. Guo, and D. Cao, "Modeling driver steering control based on stochastic model predictive control," in Proceedings of the IEEE International Conference on Systems, Man, and Cybernetics (SMC '13), pp. 3704-3709, October 2013.

[27] H. Okuda, X. Guo, Y. Tazaki, T. Suzuki, and B. Levedahl, "Model predictive driver assistance control for cooperative cruise based on hybrid system driver model," in Proceedings of the American Control Conference (ACC '14), pp. 4630-4636, June 2014. 


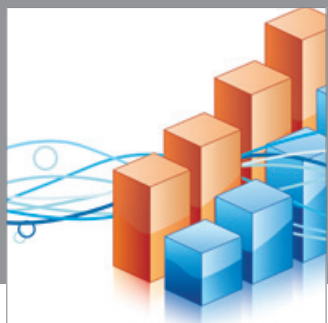

Advances in

Operations Research

mansans

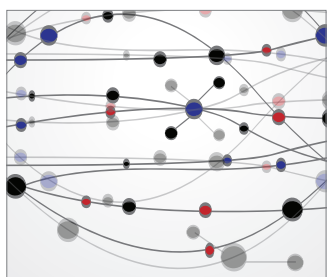

The Scientific World Journal
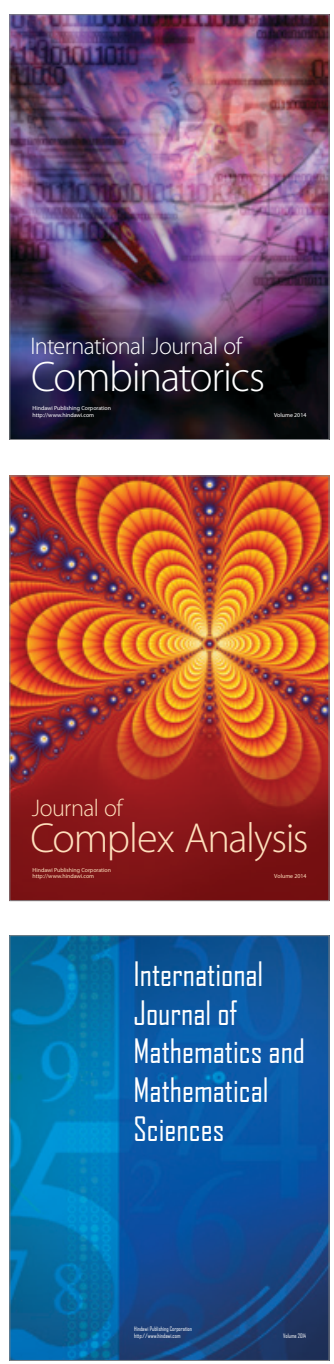
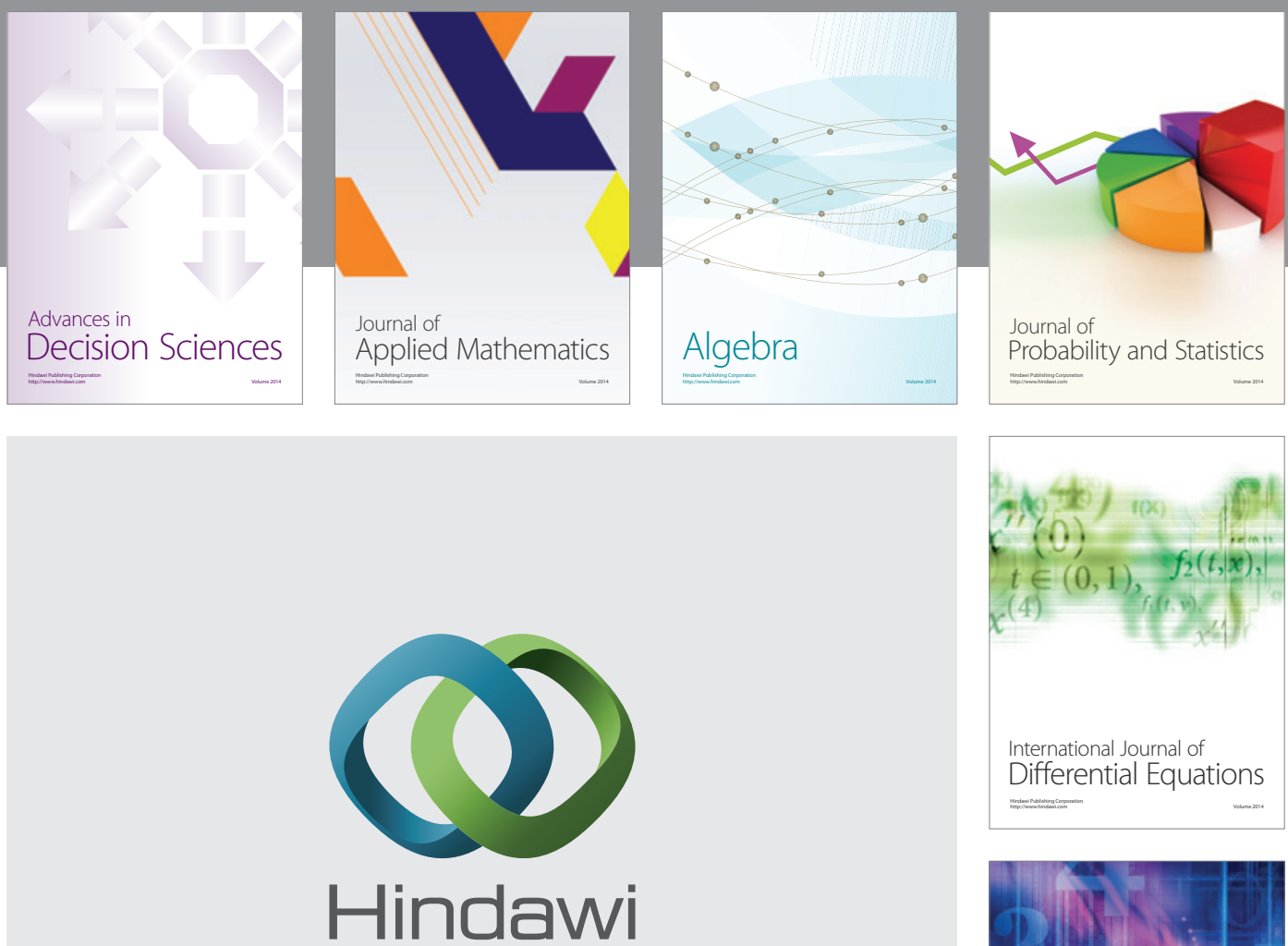

Submit your manuscripts at http://www.hindawi.com
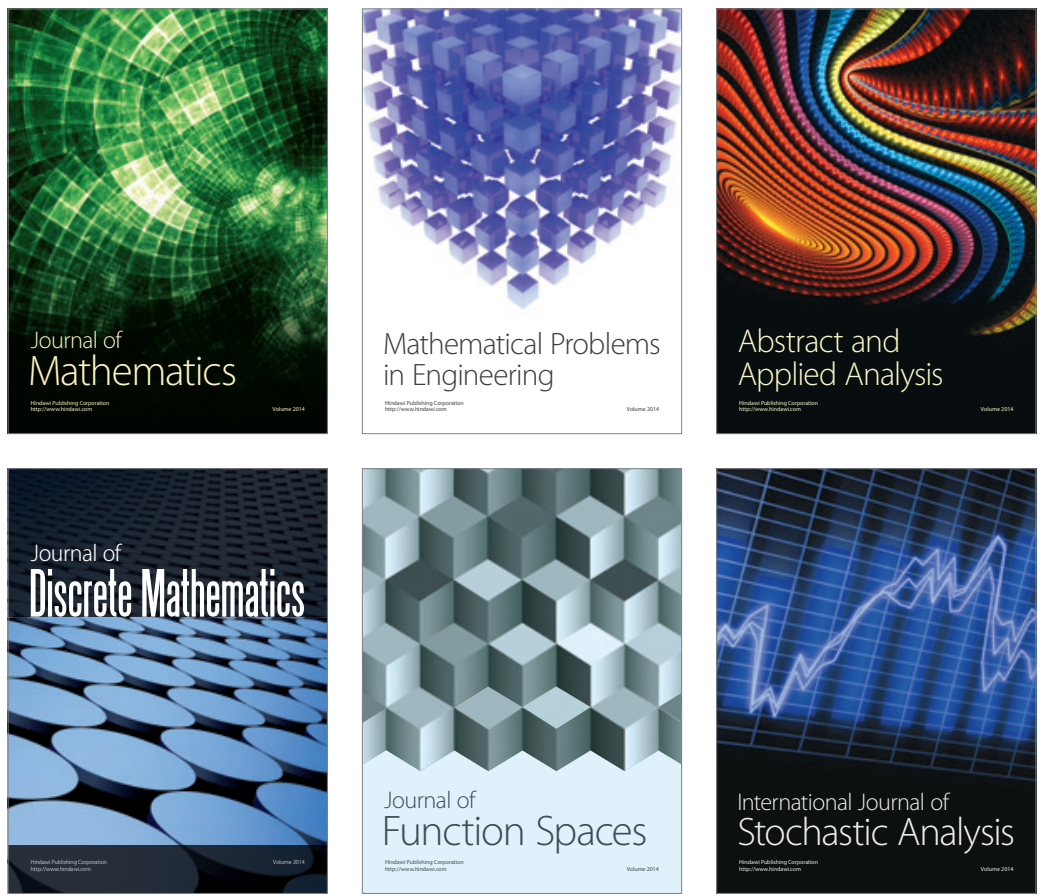

Journal of

Function Spaces

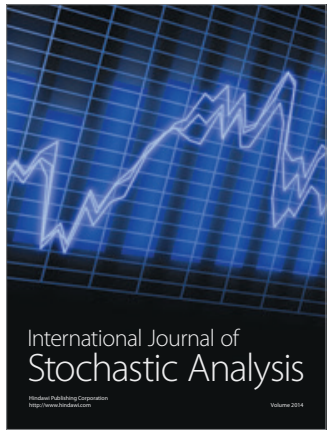

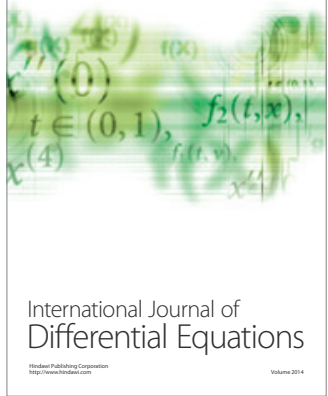
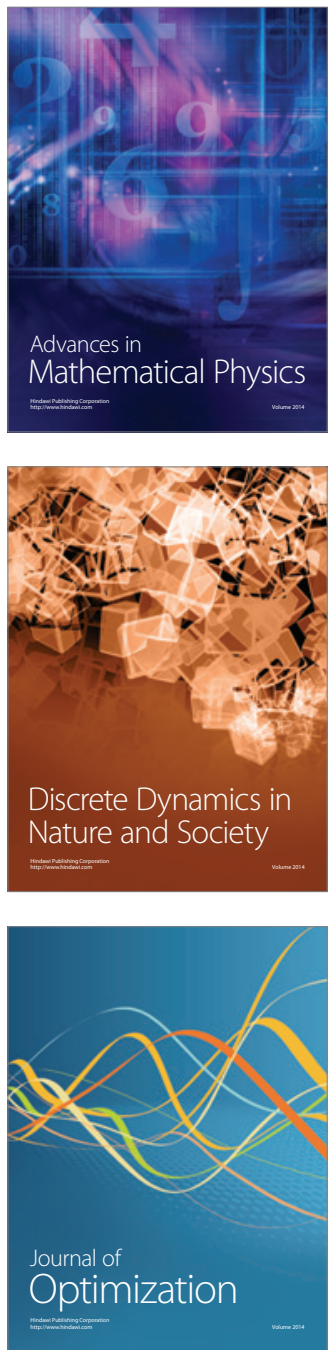